\title{
COMUNICAÇÃO BREVE Tradução e adaptação cultural para o português brasileiro da Cambridge Depersonalisation Scale (Escala de Despersonalização de Cambridge)
}

\author{
Translation and cultural adaptation to Brazilian Portuguese \\ of the Cambridge Depersonalisation Scale \\ Ana Carolina Sarquis Salgado', Leonardo Cruz de Souza², João Vinícius Salgado³, Mauricio Sierra4, \\ Antônio Lúcio Teixeira²
}

\section{RESUMO}

Objetivo: Realizar a tradução e a adaptação cultural da Escala de Despersonalização de Cambridge (EDC) para o português brasileiro. Métodos: Foi realizada a tradução da escala em duas versões, seguida por retrotradução por nativo de língua inglesa. Os itens da escala foram comparados e adaptados para o contexto brasileiro e, então, transformados em uma versão-teste, que foi aplicada em 30 participantes sem diagnóstico de transtorno psiquiátrico.

\section{Palavras-chave \\ Despersonalização, desrealização, Escala de Despersonalização de Cambridge, transtornos psiquiátricos.}

\section{Keywords}

Depersonalization, derealization, Cambridge Depersonalization Scale, psychiatric disorders.
Resultados: A versão em português da EDC mostrou boa compreensibilidade. A maior parte dos participantes relatou experiências transitórias ligadas à despersonalização nos últimos seis meses (53,3\% dos casos), incluindo a sensação de ser um observador externo de si mesmo (35\%) e sensação de estar fora do corpo (10\%). Conclusões: $O$ presente estudo confirma a alta frequência de experiências de despersonalização. Serão necessários estudos para verificação das propriedades psicométricas da escala.

\section{ABSTRACT}

Objective: To perform the translation and cultural adaptation of the Cambridge Depersonalization Scale (CDS) to Brazilian Portuguese. Methods: The scale translation was performed in two versions, followed by back translation by a native English speaker. Scale items were compared and adapted to the Brazilian context, and then transformed into a test version, applied to 30 participants without diagnosis of psychiatric disorders. Results: The Portuguese version of the CDS showed good comprehensibility. Most participants reported depersonalization transient experiences over the last six months (53.3\% of the cases), including feeling of being an outside observer of the self (35\%) and feeling of being outside the body (10\%). Conclusions: The current study confirms the high frequency of depersonalization experiences. Further studies are needed to verify the psychometric properties of the scale.

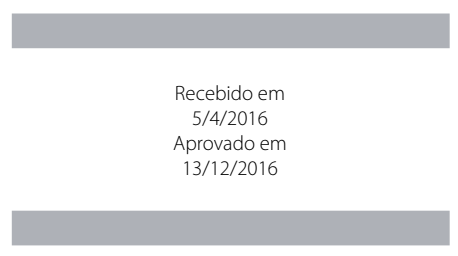

DOI: $10.1590 / 0047-2085000000141$
1 Ciências Médicas de Minas Gerais (CMMG), Faculdade de Medicina.

2 Universidade Federal de Minas Gerais (UFMG), Faculdade de Medicina.

3 Universidade Federal de Minas Gerais (UFMG), Instituto de Ciências Biológicas.

4 Escuela de Pensamiento Creativo, La Colegiatura Colombiana.

Endereço para correspondência: Ana Carolina Sarquis Salgado

Avenida do Contorno, 5491/401

30110-035 - Belo Horizonte, MG, Brasil

E-mail:acssalgado@gmail.com 


\section{INTRODUÇÃO}

A despersonalização - fenômeno relativamente comum, afetando de modo clinicamente significativo entre 1\% e 2\% da população ${ }^{1-4}$ - pode ser descrita como o estranhamento ou distanciamento de si próprio frequentemente associado à sensação de ser um observador externo dos próprios processos mentais, do corpo ou de partes do corpo ${ }^{1,5}$. São usuais as vivências complementares de anestesia sensorial e de ausência de controle sobre as próprias ações, sendo mantido intacto o senso de realidade. A desrealização, por vezes simultânea à despersonalização, diz respeito à percepção de alterações subjetivas no ambiente, descrito como irreal, distante ou artificial.

Há uma tendência atual em se entender o fenômeno da despersonalização (que inclui despersonalização, desrealização, experiências corporais anômalas) como parte de um espectro, que incluiria desde a ocorrência de experiências transitórias e inespecíficas, os sintomas de despersonalização presentes nos transtornos fóbico-ansiosos, do humor e na esquizofrenia, dentre outros, e teria seu expoente máximo em termos de gravidade no transtorno específico de despersonalização e desrealização ${ }^{1,2,5}$. Nesse transtorno, os sintomas são intensos e provocam grande sofrimento subjetivo. Os critérios diagnósticos diferem entre a CID-10 e o DSM-5 5,7 , sendo que o primeiro inclui o transtorno no grupo de "outros transtornos neuróticos" e o último, na categoria de "transtornos dissociativos". Ambas as classificações já foram criticadas ${ }^{8,9}$.

$\mathrm{Na}$ tentativa de melhor quantificar e qualificar os fenômenos subjetivos ligados à despersonalização, foram desenvolvidos instrumentos de avaliação como escalas e questionários. A Escala de Experiências Dissociativas, traduzida para o português brasileiro em $2004^{10}$, versa sobre sintomas dissociativos em geral, sendo cinco de seus 28 itens relacionados à despersonalização. Apesar de mais específicas, a Escala de Dixon"11 e a Escala de Despersonalização de Jacobs e Bovasso ${ }^{12}$ falharam na tentativa de validar o construto da despersonalização'. Já a Escala de Despersonalização de Cambridge (EDC) ${ }^{13}$ foi elaborada a partir de uma visão fenomenológica do transtorno e se tornou o instrumento mais utilizado em clínica e pesquisa. A escala é constituída por 29 subitens que representam detalhadamente os sintomas mais comuns do transtorno, como modificações nas sensações corporais, percepção subjetiva de memória e do ambiente externo, diferentes categorias de perda de interesse e prazer e fenômenos de autoscopia. Cada subitem é quantificado em relação à frequência e à duração nos últimos seis meses e recebe pontuação entre 0 e 10. A pontuação máxima possível é de 290 pontos. Os estudos realizados em inglês e em outros idiomas sugerem pontos de corte para diagnóstico do transtorno de despersonalização e desrealização entre 59 e $71^{13-15}$.
No instrumento original, um escore igual ou acima de 70 pontos é consistente com o transtorno (sensibilidade de $75,7 \%$ e especificidade de $87,2 \%)^{13}$.

A construção da EDC despertou interesse sobre o tema e possibilitou uma expansão de pesquisas na área pela possibilidade de quantificação mais precisa da gravidade dos sintomas e avaliação seriada de resposta terapêutica. Contudo, uma das dificuldades encontradas para a pesquisa do transtorno de despersonalização e desrealização no Brasil é justamente a escassez de instrumentos confiáveis e validados em português brasileiro. Os poucos trabalhos publicados sobre o tema no país se resumem a relatos isolados de casos, nos quais muitas vezes os termos despersonalização e desrealização são empregados de modo genérico. O objetivo deste trabalho, portanto, é a elaboração da primeira versão da EDC em português brasileiro.

\section{MÉTODOS}

Na primeira fase do processo, dois dos autores do artigo com conhecimento sobre o tema e domínio da língua inglesa - realizaram traduções independentes da EDC, originando duas versões em português brasileiro. Em seguida, as duas versões foram então retrotraduzidas para o inglês, também de modo independente, por tradutor bilíngue de origem inglesa. A segunda etapa consistiu em avaliar a equivalência semântica e sintáxica do novo instrumento quando comparado à versão original, realizada por todos os autores. Os itens considerados alterados foram revistos, sendo escolhida entre as duas versões de cada subitem aquela que mais se aproximava do instrumento original, produzindo-se a versão-teste. Após a aplicação da escala em um pequeno grupo amostral para verificação global do entendimento, os itens foram revistos por um especialista em letras para uma comparação independente das versões em português e inglês.

Como modelo adicional para a elaboração da escala, foi consultada a versão validada em espanhol"1 (cortesia do prof. Mauricio Sierra).

A versão-teste foi aplicada em uma pequena amostra de estudantes da Universidade Federal de Minas Gerais (UFMG) para a verificação do entendimento. Durante uma aula do Programa de Pós-Graduação em Neurociências, os alunos foram convidados a preencher o questionário, proposta aceita por dez dos estudantes presentes. Durante a aplicação do questionário, foi observada dificuldade de entendimento de alguns subitens, especialmente 1, 4, 10 e 24. A partir desse achado, os autores realizaram pequenas modificações na estruturação das sentenças, com o objetivo de melhorar sua compreensão.

A nova versão-teste foi, então, aplicada em 30 participantes recrutados entre alunos, funcionários e colaboradores do Hospital das Clínicas da UFMG (HC/UFMG) e Instituto Raul 
Soares/FHEMIG (IRS). Os critérios de inclusão adotados foram: idade entre 18 e 65 anos e assinatura do Termo de Consentimento Livre e Esclarecido (TCLE). Foram excluídos da pesquisa participantes que apresentassem qualquer doença neurológica ou apresentassem histórico de traumatismo craniano, acidente vascular encefálico ou neurocirurgia. Adicionalmente, foi utilizado como critério de exclusão o diagnóstico atual de qualquer transtorno psiquiátrico. Os participantes da segunda etapa do estudo foram avaliados por meio do Mini International Neuropsychiatric Interview (MINI) por psiquiatra e preencheram o Inventário de Depressão de Beck (BDI) e o Inventário de Ansiedade de Beck (BAI).

Este trabalho foi aprovado nos respectivos Comitês de Ética em Pesquisa das instituições participantes (HC/UFMG e IRS/FHEMIG) e todos os indivíduos, nas duas etapas do processo, assinaram o TCLE. Foram realizadas análises descritivas, e os resultados das variáveis contínuas, como as escalas $\mathrm{BDI}$ e BAI, serão apresentados sob a forma de medianas. Foi testada a normalidade da amostra pelo teste de ShapiroWilk, sendo identificada distribuição não normal.

\section{RESULTADOS}

Os trinta participantes da etapa final do estudo possuíam entre 18 e 65 anos, escolaridade mínima de ensino fundamental completo e pertenciam majoritariamente ao sexo feminino (86,6\%). A mediana da idade foi 32 anos, e a escolaridade média, de 12 anos. Foram identificadas as seguintes medianas das pontuações nas escalas de avaliação: BDI = 5, $B A I=6$, e EDC $=13$. Não foi detectado nenhum problema adicional em relação à autoaplicação da escala EDC, sendo estabelecida a versão final.

Na Tabela 1, estão descritos os resultados obtidos por meio da análise dos itens da EDC presentes nos últimos seis meses, atentando-se para: 1) a presença do sintoma (sim/ não), independentemente da frequência; 2) a duração (minutos a horas/uma semana ou mais). Em relação às experiências de despersonalização e desrealização, verificou-se que a incidência de qualquer experiência nos últimos seis meses foi de $53,1 \%$ da amostra estudada. Alguns itens, principalmente os relacionados ao subdomínio de deafetualização (por exemplo: "as minhas atividades favoritas não são mais agradáveis"), podem ter sua incidência aumentada pela confusão com sintomas depressivos, mesmo após a exclusão de todos os participantes com transtornos de humor. Porém, observa-se frequência alta de alguns fenômenos bastante específicos do construto da despersonalização, como a sensação de ser um observador externo de si mesmo, assinalada por 35,5\% dos participantes. Destaca-se, ainda, a alta incidência do fenômeno de déjà vu, relatado por $74,2 \%$ dos entrevistados.
Tabela 1. Frequência dos sintomas da Escala de Despersonalização de Cambridge (EDC) nos últimos seis meses na amostra estudada $(n=30)$

\begin{tabular}{|c|c|c|}
\hline & $\begin{array}{l}\text { Presença } \\
\text { do sintoma } \\
(n=30)\end{array}$ & $\begin{array}{l}\text { Sintoma com } \\
\text { duração } \\
>1 \text { semana } \\
(n=30)\end{array}$ \\
\hline 1. Sentir-se estranho ou excluído do mundo & $10(32,3 \%)$ & $1(10 \%)$ \\
\hline 2. Objetos parecem rasos, como numa fotografia & $4(12,9 \%)$ & 0 \\
\hline 3. 0 corpo parece não pertencer ao indivíduo & $4(12,9 \%)$ & 0 \\
\hline 4. Não sentir medo em situações amedrontadoras & $19(61,3 \%)$ & 0 \\
\hline 5. Atividades favoritas não são mais agradáveis & $14(45,2 \%)$ & $2(14,3 \%)$ \\
\hline 6. Sensação de ser um observador externo de si mesmo & $11(35,5 \%)$ & $1(9,1 \%)$ \\
\hline 7. Sabor da comida não agrada ou desagrada & $6(20 \%)$ & 0 \\
\hline 8. Corpo muito leve, flutuando no ar & $5(16,1 \%)$ & 0 \\
\hline 9. Não sentir emoções quando chora ou sorri & $6(20 \%)$ & 0 \\
\hline 10. Sensação de não ter nenhum pensamento & $8(25,8 \%)$ & 0 \\
\hline 11. Própria voz remota e irreal & $4(12,9 \%)$ & 0 \\
\hline 12. Sentir mãos e pés ficando maiores ou menores & $2(6,5 \%)$ & 0 \\
\hline 13. Ambiente externo parece irreal & $3(10 \%)$ & $1(33,3 \%)$ \\
\hline 14. Atos recentes parecem ter ocorrido há tempos & $9(29 \%)$ & 0 \\
\hline 15. Ver-se de fora do corpo, como se num espelho & $3(10 \%)$ & 0 \\
\hline 16. Memórias pessoais sem envolvimento real & $9(29 \%)$ & $1(11,1 \%)$ \\
\hline 17. Déjà vu & $23(74,2 \%)$ & $1(4,3 \%)$ \\
\hline 18. Não sentir afeto por pessoas próximas & $5(16,1 \%)$ & 0 \\
\hline 19. Objetos parecem menores ou distantes & $3(10 \%)$ & 0 \\
\hline 20. Não sentir objetos quando os toca & 0 & 0 \\
\hline 21. Não conseguir formar imagem (rostos, lugares) & $6(20 \%)$ & 0 \\
\hline 22. Distanciamento da dor física & 0 & 0 \\
\hline 23. Sensação de estar fora do corpo & $3(10 \%)$ & 0 \\
\hline 24. Sensação de movimentação mecânica do corpo & $5(16,1 \%)$ & 0 \\
\hline 25. Cheiro não agrada ou desagrada & $4(12,9 \%)$ & 0 \\
\hline 26. Pensamentos parecem ter vida própria & $7(22,6 \%)$ & $1(14,3 \%)$ \\
\hline 27. Ter que se tocar para certificar-se do próprio corpo & 0 & 0 \\
\hline 28. Incapacidade de sentir fome ou sede & $2(6,5 \%)$ & 0 \\
\hline 29. Jamais vu & $2(6,5 \%)$ & 0 \\
\hline
\end{tabular}

\section{DISCUSSÃO}

Sabe-se que o transtorno de despersonalização e desrealização se associa a importantes prejuízos sociais, funcionais e ocupacionais ${ }^{1,4,5,9}$. Estudos anteriores estimam que a despersonalização possa ser o terceiro sintoma mais comum relatado por pacientes psiquiátricos, sendo menos frequente apenas que ansiedade e humor deprimido ${ }^{1,4,5}$, e que o transtorno de despersonalização e desrealização cursa com altos índices de comorbidades psiquiátricas, em especial transtornos depressivos $(84,8 \%)$ e ansiosos $(42,6 \%)^{16}$. Estudos anteriores mostraram que a pontuação das escalas específicas de de- 
pressão e ansiedade é um importante preditor dos sintomas de despersonalização $0^{1,8,9}$. Além disso, a presença de despersonalização em qualquer transtorno neuropsiquiátrico pode ser indicativa de maior gravidade, pior resposta ao tratamento e maior utilização dos serviços de saúde mental'1,16, o que por si só justificaria a pesquisa do sintoma.

O fato de mais da metade dos entrevistados relatar sintomas transitórios (duração de minutos a horas) de DP e DR fornece evidências adicionais da alta frequência e do caráter genérico, inespecífico e possivelmente não patológico dessas experiências transitórias na amostra estudada da população brasileira. Os números apresentados no artigo coincidem com dados da literatura internacional, que estimam a ocorrência de experiências transitórias em até $74 \%$ da população ${ }^{1-4}$.

Muitas vezes, o transtorno primário de DP e DR pode ter início com experiências sintomáticas transitórias, que gradualmente ficam mais frequentes e intensas até que, por fim, não mais remitem. O mecanismo pelo qual tais experiências se tornariam permanentes em alguns indivíduos ainda não foi elucidado, o que reforça a importância do estudo das experiências transitórias, especialmente em população sem transtornos psiquiátricos. Apesar da relevância do tema, as pesquisas sobre o tema no Brasil ainda são incipientes. Uma das possíveis explicações para esse fato seria a ausência de um instrumento específico para a avaliação do transtorno disponível em português brasileiro, lacuna que este estudo visa a preencher. Artigos recentes advogam que a maioria absoluta dos pacientes (92,7\%) com despersonalização e desrealização clinicamente significativas deseja uma abordagem específica para os seus sintomas ${ }^{16}$. A disponibilidade de uma escala objetiva de avaliação pode contribuir para o reconhecimento do transtorno.

Por fim, faz-se necessário mencionar as principais limitações deste trabalho. Trata-se de um estudo realizado com uma amostra relativamente pequena de participantes e com grande participação de mulheres $(86,6 \%)$.

A primeira versão em português brasileiro da EDC mostrou boa compreensibilidade. Estudos futuros deverão avaliar suas propriedades psicométricas.

\section{CONTRIBUIÇÕES INDIVIDUAIS}

Ana Carolina Salgado - Participou de todas as etapas de elaboração do artigo, incluindo a coleta de dados.

João Vinícius Salgado e Leonardo Cruz de Souza Colaboraram na tradução e na adaptação cultural de escala.
Mauricio Sierra - É um dos autores da escala original e revisou o manuscrito.

Antônio Lúcio Teixeira - Orientou a realização do trabalho, auxiliou na coleta de dados e aprovou a versão final para publicação.

\section{CONFLITOS DE INTERESSE}

Os autores não possuem conflitos de interesse a declarar.

\section{REFERÊNCIAS}

1. Sierra M. Depersonalization: A New Look at a Neglected Syndrome. Cambridge University Press, Cambridge; 2009.

2. Hunter ECM, Sierra M, David AS. The epidemiology of depersonalisation and derealisation: a systematic review. Soc Psychiatry Psych Epidemiol. 2004;39:9-18.

3. Lee W. Prevalence and childhood antecedents of depersonalization syndrome in a UK Birth Cohort. Soc Psychiatry Psych Epidemiol. 2012;47(2):253-61.

4. Michal M, Wiltink J, Subic-Wrana C, Zwerenz R, Tuin I, Lichy M, et al. Prevalence, correlates and predictors of depersonalization experiences in the German general population. J Nerv Ment Dis. 2009;7:499-506.

5. Sierra M, David AS. Depersonalisation: a selective impairment of self- awareness. Conscious Cogn. 2011;20(1):99-108.

6. World Health Organization: F.48.1 Depersonalization-derealization syndrome, in The ICD-10 Classification of Mental and Behavioral Disorders: Clinical Descriptions and Diagnostic Guidelines. Geneva, World Health Organization; 1992. p 171.

7. American Psychiatric Association. Manual Diagnóstico e Estatístico de Transtornos Mentais. Trad. Cláudia Dornelles; 5.ed. rev. Porto Alegre: Artmed; 2014.

8. Lemche E, Surguladze SA, Brammer MJ, Phillips ML, Sierra M, David AS, et al. Multiple clinical traits predict clinical diagnosis of depersonalization disorder: implications for DSM-V. Biol Psychiatry. 2012;1;72(1):e1-2.

9. Lemche E. Dissociable brain correlates for depression, anxiety, dissociation, and somatization in depersonalization-derealization disorder. CNS spectrums. 2016;21(1):35-42.

10. Fiszman A. A adaptação transcultural para o português do instrumento Dissociative Experiences Scale para rastrear e quantificar os fenômenos dissociativos. Rev Bras Psiquiatr. 2004;26(3):164-73.

11. Dixson JC. Depersonalization phenomena in a sample population of college students. Br J Psychiatry. 1963;109:371-5.

12. Jacobs JR, Bovasso GB. Toward the clarification of the construct of depersonalization and its association with affective and cognitive dysfunctions. J Pers Assess. 1992;59:352-65.

13. Sierra M, Berrios GE. The Cambridge Depersonalisation Scale: a new instrument for the measurement of depersonalisation. Psychiatry Res. 2000;93(2):153-64.

14. Molina Castillo JJ, Martinez J, Albert Colomer C, Berrios G, Sierra M, Luque Luque R. Cross-cultural adaptation and validation of the Cambridge DP Scale. Actas Esp Psiquiatr. 2006;34(3):185-92.

15. Mogliorini V, Dell'Erba A, Sierra M, Mosticoni S, Telesforo L, Patanè M, et al. Italian (cross cultural) adaptation and validation of the Cambridge Depersonalization Scale (CDS). Epidemiology Psych Sciences. 2012;21:221-6.

16. Michal M, Adler J, Wiltink J, Reiner I, Tschan R, Wölfling K, et al. A case series of 223 patients with depersonalization-derealization syndrome. BMC Psychiatry. 2016;16:203-14. 